\title{
Transverse Transport in Two-Dimensional Relativistic Systems with Non-Trivial Spin Textures
}

\author{
Juba Bouaziz, ${ }^{1, *}$ Hiroshi Ishida, ${ }^{2}$ Samir Lounis,,${ }^{1,3}$ and Stefan Blügel ${ }^{1}$ \\ ${ }^{1}$ Peter Grünberg Institut and Institute for Advanced Simulation, \\ Forschungszentrum Jülich \& JARA, D-52425 Jülich, Germany \\ ${ }^{2}$ College of Humanities and Sciences, Nihon University, Sakura-josui, Tokyo 156-8550, Japan \\ ${ }^{3}$ Faculty of Physics, University of Duisburg-Essen, 47053 Duisburg, Germany
}

(Dated: March 12, 2021)

\begin{abstract}
Using multiple scattering theory, we show that the generally accepted expression of transverse resistivity in magnetic systems that host skyrmions, given by the linear superposition of the ordinary (OHE), the anomalous (AHE) and the topological Hall effect (THE), is incomplete and must be amended by an additional term, the "non-collinear" Hall effect (NHE). Its angular form is determined by the magnetic texture, the spin-orbit field of the electrons, and the underlying crystal structure, allowing to disentangle the NHE from the various other Hall contributions. Its magnitude is proportional to the spin-orbit interaction strength. The NHE is an essential term required for decoding two- and three-dimensional spin textures from transport experiments.
\end{abstract}

The electronic transport is paramount in condensed matter physics. It permits the probing of electronic and magnetic properties of solids by electrical means and exhibits a plethora of exciting phenomena. One of them is the Hall effect, the response measured transversely to an electric current and a perpendicularly applied magnetic field. It manifests itself in systems with broken timereversal symmetry, either due to an external magnetic field [1] leading to the ordinary Hall effect (OHE) or due to the presence of spontaneous magnetization, the latter is known as the anomalous Hall effect (AHE) [2-4]. The topological Hall effect (THE) is an additional contribution attributed to a Berry phase [5] acquired by the electrons following adiabatically a smoothly varying noncoplanar magnetization texture [6-8]. The linear superposition of the three contributions yields the commonly accepted expression for the Hall resistivity [9-18]:

$$
\rho_{\mathrm{Tot}}^{\mathrm{H}}=\rho^{\mathrm{OHE}}+\rho^{\mathrm{AHE}}+\rho^{\mathrm{THE}},
$$

where the first term scales linearly with the applied external magnetic field, the second $\left(\rho^{\mathrm{AHE}}\right)$ is linear in terms of the magnetization, and the last term $\left(\rho^{\mathrm{THE}}\right)$ is proportional to the emergent magnetic field [6-8], that is in turn proportional to the topological skyrmion number defined by the sum of all solid angles $\boldsymbol{m}_{i} \cdot\left(\boldsymbol{m}_{j} \times \boldsymbol{m}_{k}\right)$ of the magnetic moments at three different sites $i, j, k$. Equation (1) was first introduced in the seminal work of Neubauer et al. [9], in which the connection between $\rho^{\mathrm{THE}}$ and emergence of the skyrmion lattice ( $A$ phase) in B20 MnSi was established. Since Eq. (1) allows a fully electric detection and quantification [9, 19-27] of magnetic skyrmions, (1) is of crucial importance for the characterization of skyrmions, especially compact skyrmions smaller than the resolution limit of imaging techniques.

In recent years, however, especially for magnetic films and multilayers with interfaces of heavy materials having large spin-orbit interactions, doubts have been raised about the simple relationship between the Hall resistivity and the skyrmion count [28]. For instance, a quantitative analysis on $\mathrm{Ir} / \mathrm{Fe} / \mathrm{Co} / \mathrm{Pt}$ multilayers shows much larger experimental topological-Hall resistivities than expected from the skyrmion density measured by magnetic force microscopy, which was partly explained by contributions of worm-like spin textures with a non-vanishing topological charge [23]. In addition, in the weak coupling limit of the moving electron spin to the underlying magnetic texture small vortex-like textures without topological charge are able to produce a finite topological Hall effect, which vanishes for larger spin textures [29], indicating its nontopological nature.

In this letter we evaluate the transverse resistivity of a two-dimensional (2D) ensemble of non-collinear magnetic atoms interacting through a relativistic electron gas by means of multiple scattering theory. Treating spin-orbit interaction (SOI) and non-collinear magnetism (NCM) on the same footing we demonstrate that the Hall resistivity tensor (1) needs to be amended by an additional term, the non-collinear Hall effect (NHE):

$$
\begin{aligned}
\rho^{\mathrm{NHE}} & =\sum_{i j l} \rho_{i j l}^{\mathrm{NHE}}\left[\left(\boldsymbol{D}_{i j} \cdot \boldsymbol{m}_{i}\right)\left(\boldsymbol{m}_{j} \cdot \boldsymbol{m}_{l}\right)\right. \\
& \left.+\left(\boldsymbol{D}_{i j} \cdot \boldsymbol{m}_{j}\right)\left(\boldsymbol{m}_{i} \cdot \boldsymbol{m}_{l}\right)-\left(\boldsymbol{D}_{i j} \cdot \boldsymbol{m}_{l}\right)\left(\boldsymbol{m}_{i} \cdot \boldsymbol{m}_{j}\right)\right],
\end{aligned}
$$

looming from the interference of both the SOI and the NCM. $\boldsymbol{m}_{i}$ indicates the unit vector of the magnetic moment at site $i . \quad \rho_{i j k}^{\mathrm{NHE}}$ depends like the DzyaloshinskiiMoriya interaction (DMI) [30, 31] on the spin-flip components of the relativistic Green function of the electron gas [32], and thus correlates to the SOI, depends on the electronic structure. The orientation of the unit vector $\mathcal{D}_{i j}=-\mathcal{D}_{j i}$ depends on the direction of the vector connecting the sites $i, j$, the crystal symmetry coupling orbital with spin degrees and follows the symmetry rules of the DMI.

Having the description of surfaces, films and multilayers in mind we model the electronic structure by a $2 \mathrm{D}$ electrons gas under Rashba-, Dresselhaus- or Weyl-type 
SOIs. The magnetic atoms are represented by a collection of localized spins with an $s d$-like coupling to the free-electrons [33]. The scattering off the magnetic sites is taken in the weak-coupling limit [29, 34], whereby not only adiabatic but also non-adiabatic effects on the resistivity are considered.

We show that the NHE can assume significant contributions in non-collinear magnets, that it occurs in twoand three-dimensional spin textures and that it can be used to discriminate Bloch- from Néel-type skyrmions. We suggest an experimental protocol to disentangle the NHE from other contributions at play. Considering the micromagnetic limit of (2) we recover the recently conjectured chiral Hall effect [35], and unravel its microscopic origin.

The starting point of the derivation of (2) is the multiple scattering theory applied to a collection of sites forming a complex magnetic configuration as of a nanoskyrmion shown in Fig. 2a. An incident free-electron in the state $|\boldsymbol{k} \sigma\rangle$ with an energy $\mathcal{E}_{\boldsymbol{k}}=\hbar k^{2} / 2 m$ ( $k$ and $m$ being the wave number and electron mass, respectively) carrying a spin $\sigma$ scatters elastically at a collection of localized potentials [36, 37] into a state $\left|\boldsymbol{k}^{\prime} \sigma^{\prime}\right\rangle$ with $k=k^{\prime}$, and $\boldsymbol{k}$ and $\boldsymbol{k}^{\prime}$ including a scattering angle $\phi=\angle \boldsymbol{k} \boldsymbol{k}^{\prime}$. In the far-field limit, the resulting wave function consists of a linear superposition of the incoming wave and the outgoing scattered one given by $[29,34,37]$ :

$$
\psi_{\boldsymbol{k} \sigma}(\vec{r})=e^{i \boldsymbol{k r}}|\sigma\rangle+\sum_{\sigma^{\prime}} \frac{e^{i k r}}{\sqrt{r}} f_{k, \sigma^{\prime} \sigma}(\phi)\left|\sigma^{\prime}\right\rangle .
$$

$|\sigma\rangle$ is the eigenstate of $\hat{S}_{z} \cdot f_{k, \sigma^{\prime} \sigma}(\phi)$ represents the scattering amplitude, a central quantity within our scattering approach, which is defined via the differential cross section

$$
\frac{d \Sigma^{\mathrm{H}}}{d \phi}=\sum_{\sigma \sigma^{\prime}}\left|f_{k, \sigma^{\prime} \sigma}(\phi)\right|^{2}
$$

directly related to the Hall current $\mathcal{J}_{\mathrm{H}}=k \Sigma^{\mathrm{H}}$ (see Supplementary Material [38]). Following standard scattering theory, $f_{k, \sigma^{\prime} \sigma}(\phi)$ is evaluated by the LippmannSchwinger equation [34, 37] employing the relativistic Green function of the propagating electron, and including the multiple scattering events experienced by the incident electron within the skyrmion via the scattering matrix $\mathcal{T}$. In the weak coupling regime $[29,34]$, the $(\mathcal{T})$ matrix is computed using the second Born approximation. Inside the skyrmion, the electron propagates under a spin-orbit field in a structure asymmetric environment $[39,40]$. This approach allows to establish a transparent relation between the scattering cross section and the orientation of the magnetic moments in the presence of relativistic effects (as shown in Supplementary Material [38]). The presence of the combined effect of SOI and NCM results in a finite asymmetric (right/left) scattering in the transverse direction, i.e. a finite Hall effect.
The Hall current $\mathcal{J}_{\mathrm{H}}$ is then tied back to the resistivity employing the Boltzmann equation (see Supplementary Material [38]). Finally, the electric current is considered with respect to the limit of small external electric fields. Since in this case the electron transport is dominated by the Fermi surface contributions [41], the current is calculated at the Fermi energy.

In order to illustrate and identify the contribution of the NHE to the Hall signal, we consider a magnetic trimer, which is the smallest nano-structure capable of generating a finite scalar spin chirality [40, 42]. The three magnetic atoms form an equilateral triangle separated by a distance of $d=3 \AA$. The Fermi energy is set to $\varepsilon_{\mathrm{F}}=0.8 \mathrm{eV}$, which coincides with the Fermi energy at metallic surfaces [43]. We consider the weak scattering limit by setting the spin-dependent scattering phase shift to $\delta^{\uparrow}=\frac{7 \pi}{8}$ and $\delta^{\downarrow}=\frac{5 \pi}{8}$ for spin-up and -down electrons, respectively. The evolution of the outgoing Hall current as function of spin non-collinearity expressed in terms of the opening angle of the magnetic moments $\theta_{\mathrm{M}}$, and the spin-orbit field of the electrons described here by the Rashba-model with a spin-orbit strength expressed by the ratio $\mathcal{R}=k_{\mathrm{so}} / k_{\mathrm{F}}$ of the spin-orbit wave vector $k_{\mathrm{so}}$ and the Fermi wave vector $k_{\mathrm{F}}$ is depicted in Fig. 1. The associated azimuthal angle $\phi_{\mathrm{M}}$ of the moments at each corner of the triangle changes from corner to corner by $120^{\circ}$, i.e. $\phi_{\mathrm{M}}=\{\pi / 2,7 \pi / 6,11 \pi / 6\}$. Three different quantities are shown: the THE, the NHE and their sum. The value of $\mathcal{R}$ can be engineered to reach rather large magnitudes by tuning the Rashba spin splitting $\propto k_{\text {so }}[44-46]$.

The THE contribution shown in Fig. 1a follows the scalar spin chirality $\chi_{i j k}=\boldsymbol{m}_{i} \cdot\left(\boldsymbol{m}_{j} \times \boldsymbol{m}_{k}\right)$, it maximises at $\theta_{\mathrm{M}}=60^{\circ}, 120^{\circ}$ and vanishes at the ferromagnetic $\left(\theta_{\mathrm{M}}=0^{\circ}, 180^{\circ}\right)$ and coplanar $\left(\theta_{\mathrm{M}}=90^{\circ}\right)$ spin configuration. Interestingly, the THE current is altered by the SOI as it decreases for larger values of $\mathcal{R}$, a closer inspection of $\mathcal{J}_{\text {THE }}$ shows that it depends only on the spin-conserving components of the Rashba Green function. In the limit $\mathcal{R} \ll 1$, the latter decreases quadratically upon increasing spin-orbit strength. On the other hand, the NHE shown in Fig. 1b reaches its highest value when $\theta_{\mathrm{M}}=90^{\circ}$. This can be attributed to a larger scalar product $\left(\mathcal{D}_{i j} \cdot \boldsymbol{m}_{i}\right)$ since $\mathcal{D}_{i j}$ is locked inside the $(x y)$-plane. It also results in a symmetric signal when $m_{z} \rightarrow-m_{z}$. The strength of the NHE increases linearly for small values of $\mathcal{R}$, and is determined by the spin off-diagonal components of the Green function (Supplementary Material [38]), similarly to the DMI [32].

In order to experimentally distinguish between the THE and NHE we make use of the observation that by including the NHE in the overall picture, the Hall signal becomes asymmetric when $\theta_{\mathrm{M}} \rightarrow \pi-\theta_{\mathrm{M}}$ (see Fig. 1c). Thus, considering a Hall setup for a skyrmion system, two sets of measurements with opposite orientations of the major axis of the crystal should be performed. The 

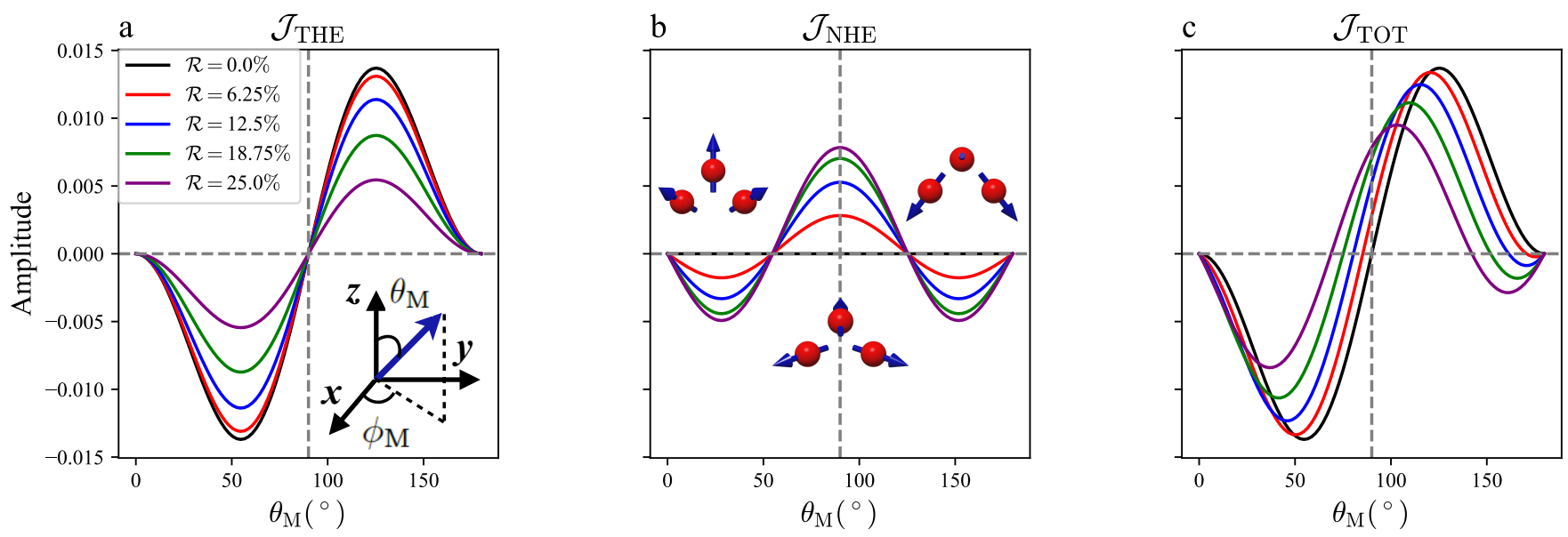

FIG. 1. Evolution of the scattered current for a magnetic trimer in an equilateral geometry as a function of the opening angle $\theta_{\mathrm{M}}$ of the magnetic moments and of the spin-orbit strength $\mathcal{R}$. a) The THE contribution to the current determined by the scalar spin chirality. b) NHE current originating from relativistic effects, its non-trivial angular dependence is given in Eq. (2). c) Total Hall current emerging from three spin processes, which present an non-symmetric dependence for $\theta_{\mathrm{M}} \rightarrow \pi-\theta_{\mathrm{M}}$.

anti-symmetric part of the signal is the THE, while the symmetric one leads to the NHE.

A remarkable property of the NHE is its dependence on the crystal structure, which underlines its deep relationship with the SOI. The vector $\mathcal{D}_{i j}$ provides a direct connection between the electronic structure of the material and the NHE (see Supplementary Material [38]). Therefore, Eq. (2) can be expressed for the well-known 2D spin-orbit Hamiltonians. The different expressions of $\mathcal{D}_{i j}^{2 \mathrm{D}}$ for the Rashba [39], Dresselhaus [47] and Weyl Hamiltonians [48] are given in Table I. This offers an additional perspective for the engineering of the functional dependence of the NHE on the magnetization direction using the symmetry properties of the electronic band structure (crystal structure), besides tuning its magnitude by changing the coupling parameter as shown in Fig. 1. Note also that once the NHE can be analyzed separately from the overall Hall response, it could be used as a means to study the band structure and spin momentum locking based on all-electric measurements. The relation between the NHE and the crystal structure is an illustration of Neumann's principle, which dictates that the point group symmetry of a system is reflected in its physical quantities [49-51].

After expanding Eq. (2) in terms of the components of the magnetization, and plugging in the various expressions of $\mathcal{D}_{i j}^{2 \text { D }}$ provided in Table I, one yields an expression of the non-collinear Hall resistance:

$$
\rho_{2 \mathrm{D}}^{\mathrm{NHE}}=\sum_{i j l} \rho_{i j l}^{2 \mathrm{D}} \mathcal{F}_{i j l}(\boldsymbol{m})\left(\underline{\mathcal{S}}^{2 \mathrm{D}} \cdot \hat{\boldsymbol{r}}_{i j}\right)
$$

$\mathcal{F}_{i j k}(\boldsymbol{m})$ contains the angular forms of the magnetization (for details see Supplementary Material [38]). $\hat{\boldsymbol{r}}_{i j}=$ $\left(\boldsymbol{r}_{i}-\boldsymbol{r}_{j}\right) /\left|\boldsymbol{r}_{i}-\boldsymbol{r}_{j}\right|, \boldsymbol{r}_{i}$ defining the position of the magnetic site $i$. The matrix $\underline{\mathcal{S}}^{2 \mathrm{D}}$ reflects the symmetry of the
TABLE I. Generic Rashba, Dresselhaus and Weyl Hamiltonians in $\boldsymbol{k}$-space, and their corresponding expressions for $\boldsymbol{D}_{i j}$ defining the NHE resistivity. $\phi_{i j}$ is the angle between the $\boldsymbol{r}_{i j}$ (bond vector connecting $i$ to $j$ ) and the $x$-axis (see Supplementary Material [38]).

\begin{tabular}{lcc}
\hline \hline System & Hamiltonian & $\mathcal{D}_{i j}$ \\
\hline Rashba & $\alpha\left(k_{y} \boldsymbol{\sigma}_{x}-\boldsymbol{\sigma}_{y} k_{x}\right)$ & $\left(\sin \phi_{i j},-\cos \phi_{i j}\right)$ \\
Dresselhaus & $\beta\left(k_{y} \boldsymbol{\sigma}_{y}-\boldsymbol{\sigma}_{x} k_{x}\right)$ & $\left(-\cos \phi_{i j}, \sin \phi_{i j}\right)$ \\
Weyl & $\gamma\left(k_{y} \boldsymbol{\sigma}_{y}+\boldsymbol{\sigma}_{x} k_{x}\right)$ & $\left(\cos \phi_{i j}, \sin \phi_{i j}\right)$ \\
\hline \hline
\end{tabular}

underlying spin-orbit model as:

$$
\underline{\mathcal{S}}^{\mathrm{R}}=i \boldsymbol{\sigma}_{y}, \quad \underline{\mathcal{S}}^{\mathrm{D}}=-\boldsymbol{\sigma}_{z}, \quad \underline{\mathcal{S}}^{\mathrm{W}}=\boldsymbol{\sigma}_{0},
$$

where $\boldsymbol{\sigma}$ are the conventional Pauli matrices, and $\boldsymbol{\sigma}_{0}$ is the unity matrix. R, D and W stand for Rashba, Dresselhaus and Weyl, respectively. For the Rashba and Dresselhaus Hamiltonians describing polar systems the form of $\underline{\mathcal{S}}^{2 \mathrm{D}}$ is anti-symmetric, while it has a symmetric form for the Weyl Hamiltonian. A similar behavior was also identified for the DMI in Refs. [52-54].

We turn now to the discussion of the signature of complex magnetic textures [55] on the NHE. Considered are: Néel- and Bloch-type skyrmions [56], antiskyrmions, merons [57], and higher-order skyrmions [55]. These topological entities are described by the topological charge $(Q=-m)$, the vorticity $(m)$ and helicity $(\gamma)$ relating the azimuthal angle of the magnetization, $\Phi=m \phi_{\mathrm{M}}+\gamma$, to azimuthal angle $\phi_{\mathrm{M}}$ of the lattice [56]. We consider skyrmions with a small radius of $R_{\mathrm{sk}}=1.5 \mathrm{~nm}$, as typical for skyrmions created in magnetic transition-metal monolayers at heavy metal interfaces, e.g. $\mathrm{Pd} / \mathrm{Fe} / \mathrm{Ir}(111)$ [58-60].

Fig. 2 depicts the topological and non-collinear Hall currents for six different spin textures in systems with 

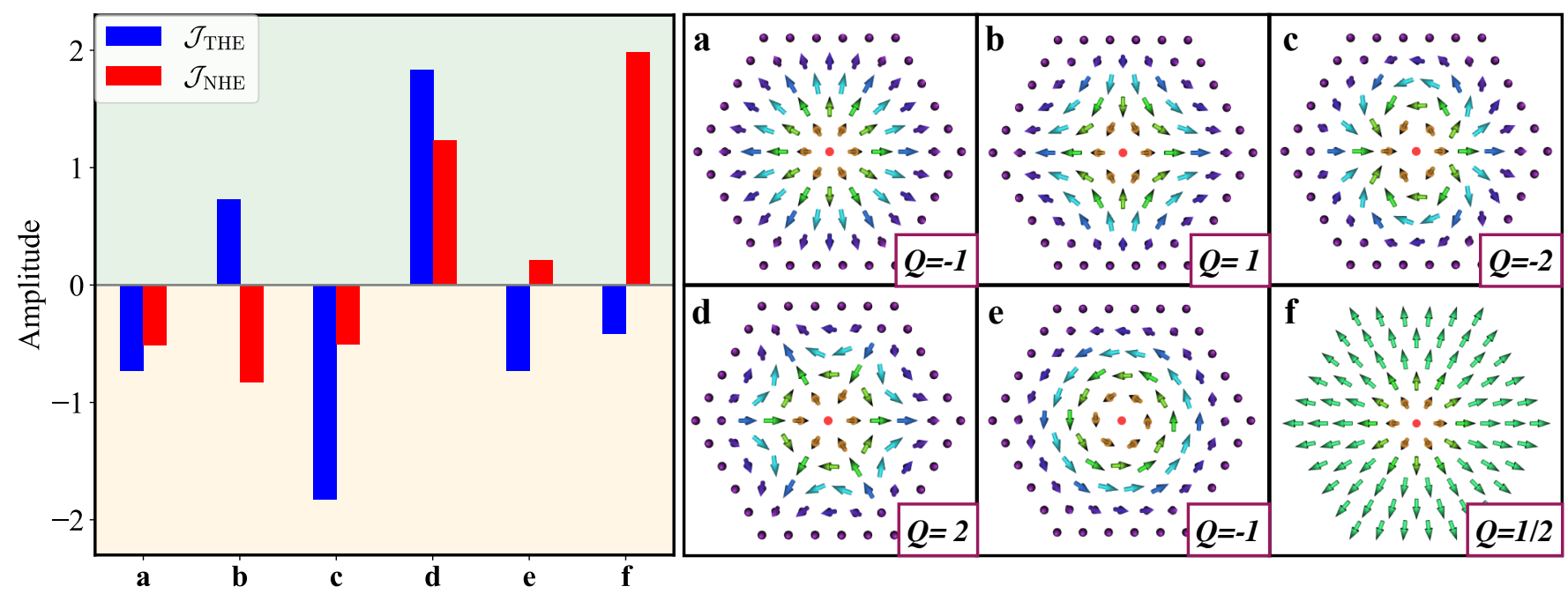

FIG. 2. The chart in the left panel describes the size of the topological (blue) and non-collinear (red) Hall current for six topological spin textures calculated for Rashba-type SOC with spin-orbit strength of $\mathcal{R}=25 \%$. The latter are shown in the right panel: a) Néel-type skyrmion, b) anti-skyrmion, c) bi-skyrmion, d) anti-bi-skyrmion, e) Bloch-type skyrmion, f) meron. We consider clusters containing 91 magnetic sites with a nearest neighbor distance of $d=3 \AA$. smearing $\Gamma=0.2 \mathrm{eV}$ is used in the vicinity of the Fermi energy to account for eventual temperature or disorder effects.

Rashba spin-orbit field (the results for the Dresselhaus and Weyl Hamiltonians are shown in Supplementary Fig. 3). Since in the adiabatic limit of transport the THE contribution is proportional to the topological charge, Néel- (a) and Bloch-type (e) skyrmions exhibit the same value of $\mathcal{J}_{\text {THE }}[29]$. The same holds true for the other spin textures, the anti-skyrmion (b) has the opposite charge and subsequently the opposite sign of $\mathcal{J}_{\text {THE }}$, the bi-skyrmion has twice the charge and doubles the magnitude of the current.

On the other hand, the NHE is found to be nonnegligible, and even colossal in some cases without any apparent connection to the spin chirality or the underlying topology of the magnetic texture. It is also notable that the NHE acquires a different value for the Néel (a) and Bloch (e) skyrmions, enabling to discriminate the two species. This difference is due to the NHE being a relativistic effect breaking the $\mathcal{S U}(2)$ rotation symmetry.

A pattern emerges from Fig. 2, the NHE is large for textures $b$ and $d$, hence it is increased by a negative vorticity $(m<0)$. At the same time, it is notably reduced for the Bloch skyrmion (e), suggesting that it diminishes in the presence of a finite helicity $\gamma \neq 0$. The latter argues in favor of the NHE being rather weak or insignificant in bulk systems with Bloch skyrmions such as MnSi. Finally, as observed in Fig. 1b the NHE is largest when spins are locked in-plane. Thus, the drastic increase of the NHE comparing the meron (f) to the Néel skyrmion (a) can be inferred to a larger number of magnetic sites lying within the $(x y)$-plane containing the electron gas.

In the limit of a slowly varying magnetic texture, i.e. in the adiabatic limit, the Hall resistivity can be expressed in terms of the magnetization density and its gradients [7,61]. Taking this limit and expanding Eq. (2) to second order in the magnetization gradients, we provide a general micromagnetic form of the Hall resistivity, which incorporates new anisotropic contributions due to the SOI (see Supplementary Material [38]):

$$
\begin{aligned}
\rho^{\mathrm{H}} & =\sum_{\alpha \neq \beta} \rho_{\alpha \beta}^{\mathrm{THE}} \boldsymbol{m} \cdot\left(\partial_{\alpha} \boldsymbol{m} \times \partial_{\beta} \boldsymbol{m}\right) \\
& +\sum_{i j l} \rho_{i j l}^{\mathrm{NHE}}\left(\boldsymbol{m} \cdot \boldsymbol{D}_{i j}\right)+\sum_{\alpha \beta} \rho_{1, \alpha \beta}^{\mathrm{NHE}} \partial_{\alpha} m^{\beta} \\
& +\sum_{\alpha \beta \gamma} \rho_{2, \alpha \beta \gamma}^{\mathrm{NHE}} \partial_{\alpha} \partial_{\beta} m^{\gamma}+\sum_{\alpha \beta \gamma} \rho_{3, \alpha \beta \gamma}^{\mathrm{NHE}}\left(\partial_{\alpha} \boldsymbol{m}\right)\left(\partial_{\beta} \boldsymbol{m}\right) m^{\gamma}
\end{aligned}
$$

with $\alpha, \beta, \gamma \in\{x, y, z\}$. The first term on the right-hand side consists of the topological charge density known from the THE $[7,61]$. Four additional terms are derived: The second term is linear in the magnetization, nonetheless, it originates from a three-site scattering process. It leads to an antisymmetric contribution to the Planar Hall effect, which has been measured in the Heusler alloy $\mathrm{Fe}_{3} \mathrm{Si}$ [62]. The term linear in the gradient of the magnetization we identify as the recently predicted chiral Hall effect [35]. The fourth contribution involves the curvature of $\boldsymbol{m}$. It is expected to be a non-negligible component for strongly inhomogeneous magnetization fields or when $\boldsymbol{m}$ is constricted in curved geometries [63, 64]. Finally, the last element of Eq. (7) has a form similar to the THE as it involves the magnetization and a product of its gradients. Which components $\{\alpha, \beta, \gamma\}$ contribute are purely determined by the SOI-dependent form $\mathcal{D}_{i j}$. In analogy, similar higher order gradient/anisotropic corrections may also emerge at the level of the orbital magnetisation in 
presence of chiral spin textures [65].

The result of Eq. (7) is another important outcome of this letter. It displays in a clear fashion that when relativistic effects are accounted for (even in the adiabatic limit), no topological spin texture is necessary to generate a non-conventional Hall effect, i.e. a Hall effect that is neither proportional to the magnetization nor to the external field. The derived formula incorporates various terms of different orders that could potentially compete or collectively contribute to the Hall signal. As a result, this renders the one-to-one correspondence between the spin texture and Hall measurement rather complex and intricate.

To conclude, we derived contributions to the transverse resistivity emerging from three-site scattering processes at magnetic atoms due to electrons subject to SOI and structure inversion asymmetry and showed that the linear superposition of the OHE, AHE, and THE (Eq. (1)) must be extended by a new contribution, the non-collinear Hall effect (NHE). The new Hall effect has far-reaching consequences, e.g.: (i) Its magnitude and angular form can be engineered by tuning the electronic band structure. (ii) It can give rise to substantial Hall responses in compensated magnets, which we conjecture is the reason for the substantial Hall response observed in the kagome magnets $\mathrm{Mn}_{3} \mathrm{Sn}$ and $\mathrm{Mn}_{3} \mathrm{Ge}$ [66, 67], where the NHE can also be easily disentangled from the THE since non-coplanar spins are located in the atomic plane. (iii) It can resolve the nature of topological spin-textures as our comparison to the THE showed. Finally, in the micromagnetic limit, the NHE translates into a superposition of different terms, including contributions proportional to the curvature of the magnetization, which could be of particular interest in systems hosting magnetic Hopfions [64, 68]. All these findings open a new vista for the analysis of Hall signals of non-collinear magnets and magnets with complex spin-textures as well as their characterization through Hall experiments.

\section{ACKNOWLEDGMENTS}

We are grateful to Fabian Lux and Yuriy Mokrousov for enlightening discussions on the different Hall contributions. We gratefully acknowledge financial support from the DARPA TEE program through grant MIPR (\# HR0011831554) from DOI, from the European Research Council (ERC) under the European Union's Horizon 2020 research and innovation program (Grant No. 856538, project "3D MAGiC" and ERC-consolidator grant 681405 - DYNASORE), from Deutsche Forschungsgemeinschaft (DFG) through SPP 2137 "Skyrmionics" (Project BL 444/16) and the Collaborative Research Centers SFB 1238 (Project C01), respectively. *j.bouaziz@fz-juelich.de

[1] E. H. Hall, "On a new action of the magnet on electric currents," American Journal of Mathematics 2, 287-292 (1879).

[2] J. M. Luttinger, "Theory of the Hall Effect in Ferromagnetic Substances," Phys. Rev. 112, 739-751 (1958).

[3] Naoto Nagaosa, "Anomalous Hall Effect-A New Perspective-," Journal of the Physical Society of Japan 75, 042001-042001 (2006).

[4] N. Nagaosa, J. Sinova, S. Onoda, A. H. MacDonald, and N. P. Ong, "Anomalous Hall effect," Rev. Mod. Phys. 82, 1539-1592 (2010).

[5] M. V. Berry, "Quantal phase factors accompanying adiabatic changes," Proceedings of the Royal Society of London. A. Mathematical and Physical Sciences 392, 45-57 (1984).

[6] Y. Taguchi, Y. Oohara, H. Yoshizawa, N. Nagaosa, and Y. Tokura, "Spin Chirality, Berry Phase, and Anomalous Hall Effect in a Frustrated Ferromagnet," Science 291, 2573-2576 (2001).

[7] P. Bruno, V. K. Dugaev, and M. Taillefumier, "Topological Hall Effect and Berry Phase in Magnetic Nanostructures," Phys. Rev. Lett. 93, 096806 (2004).

[8] G. Tatara, H. Kohno, J. Shibata, Y. Lemaho, and K-J. Lee, "Spin torque and force due to current for general spin textures," Journal of the Physical Society of Japan 76, 054707-054707 (2007).

[9] A. Neubauer, C. Pfleiderer, B. Binz, A. Rosch, R. Ritz, P. G. Niklowitz, and P. Böni, "Topological Hall Effect in the A Phase of MnSi," Phys. Rev. Lett. 102, 186602 (2009).

[10] M. Lee, W. Kang, Y. Onose, Y. Tokura, and N. P. Ong, "Unusual Hall effect anomaly in MnSi under pressure," Phys. Rev. Lett 102, 186601 (2009).

[11] N. Kanazawa, Y. Onose, T. Arima, D. Okuyama, K. Ohoyama, S. Wakimoto, K. Kakurai, S. Ishiwata, and Y. Tokura, "Large topological Hall effect in a shortperiod helimagnet MnGe," Phys. Rev. Lett 106, 156603 (2011).

[12] Yufan Li, N Kanazawa, XZ Yu, A Tsukazaki, M Kawasaki, M Ichikawa, XF Jin, F Kagawa, and Y Tokura, "Robust formation of skyrmions and topological Hall effect anomaly in epitaxial thin films of MnSi," Phys. Rev. Lett 110, 117202 (2013).

[13] Keita Hamamoto, Motohiko Ezawa, and Naoto Nagaosa, "Quantized topological Hall effect in skyrmion crystal," Phys. Rev. B 92, 115417 (2015).

[14] G. Yin, Y. Liu, Y. Barlas, J. Zang, and R. K. Lake, "Topological spin Hall effect resulting from magnetic skyrmions," Phys. Rev. B 92, 024411 (2015).

[15] J. L Lado and J. Fernández-Rossier, "Quantum anomalous Hall effect in graphene coupled to skyrmions," Phys. Rev. B 92, 115433 (2015).

[16] B. Göbel, Alexander Mook, Jürgen Henk, and Ingrid Mertig, "Unconventional topological Hall effect in skyrmion crystals caused by the topology of the lattice," Physical Review B 95, 094413 (2017).

[17] B. Göbel, A. Mook, J. Henk, and I. Mertig, "The family of topological Hall effects for electrons in skyrmion crystals," The European Physical Journal B 91, 179 (2018). 
[18] K. Nakazawa, M. Bibes, and H. Kohno, "Topological Hall effect from strong to weak coupling," Journal of the Physical Society of Japan 87, 033705 (2018).

[19] T. Schulz, R. Ritz, A. Bauer, M. Halder, M. Wagner, C. Franz, C. Pfleiderer, K. Everschor, M. Garst, and A. Rosch, "Emergent electrodynamics of skyrmions in a chiral magnet," Nature Physics 8, 301-304 (2012).

[20] K-Y. Meng, A. S. Ahmed, M. Baćani, A-O. Mandru, X. Zhao, N. Bagués, B. D Esser, J. Flores, D. W. McComb, H. J. Hug, and Y. Fengyuan, "Observation of nanoscale skyrmions in $\mathrm{SrIrO} 3 / \mathrm{SrRuO} 3$ bilayers," Nano letters 19, 3169-3175 (2019).

[21] A. Soumyanarayanan, M. Raju, A.L. G. Oyarce, A. K.C. Tan, M-Y. Im, A. P Petrović, P. Ho, K.H. Khoo, M. Tran, C.K. Gan, F. Ernult, and C. Panagopoulos, "Tunable room-temperature magnetic skyrmions in $\mathrm{Ir} / \mathrm{Fe} / \mathrm{Co} / \mathrm{Pt}$ multilayers," Nature Materials 16, 898-904 (2017).

[22] C. S. Spencer, J. Gayles, N. A. Porter, S. Sugimoto, Z. Aslam, C. J. Kinane, T. R. Charlton, F. Freimuth, S. Chadov, S. Langridge, J. Sinova, C. Felser, S. Blügel, Y. Mokrousov, and C. H. Marrows, "Helical magnetic structure and the anomalous and topological Hall effects in epitaxial B20 $\mathrm{Fe}_{1-y} \mathrm{Co}_{y}$ Ge films," Phys. Rev. B 97, 214406 (2018).

[23] M. Raju, A. Yagil, A. Soumyanarayanan, A. K.C. Tan, A. Almoalem, F. Ma, O.M. Auslaender, and C. Panagopoulos, "The evolution of skyrmions in $\mathrm{Ir} / \mathrm{Fe} / \mathrm{Co} / \mathrm{Pt}$ multilayers and their topological Hall signature," Nature Communications 10, 1-7 (2019).

[24] D. Liang, J. P. DeGrave, M. J. Stolt, Y. Tokura, and S. Jin, "Current-driven dynamics of skyrmions stabilized in MnSi nanowires revealed by topological Hall effect," Nature Communications 6, 1-8 (2015).

[25] N. Kanazawa, Y. Onose, T. Arima, D. Okuyama, K. Ohoyama, S. Wakimoto, K. Kakurai, S. Ishiwata, and Y. Tokura, "Large topological Hall effect in a shortperiod helimagnet MnGe," Phys. Rev. Lett 106, 156603 (2011).

[26] J. Jiang, Di. Xiao, F. Wang, J-H. Shin, D. Andreoli, J. Zhang, R. Xiao, Y-F. Zhao, M. Kayyalha, L. Zhang, K. Wang, Z. Jiadong, L. Chaoxing, S. Nitin, Moses H. W. C., and C-Z. Chang, "Concurrence of quantum anomalous Hall and topological Hall effects in magnetic topological insulator sandwich heterostructures," Nature Materials , 1-6 (2020).

[27] B.M. Ludbrook, G. Dubuis, A-H. Puichaud, B.J. Ruck, and S. Granville, "Nucleation and annihilation of skyrmions in $\mathrm{Mn} 2 \mathrm{CoAl}$ observed through the topological Hall effect," Scientific reports 7, 1-8 (2017).

[28] Lena Wysocki, Lin Yang, Felix Gunkel, Regina Dittmann, Paul H. M. van Loosdrecht, and Ionela Lindfors-Vrejoiu, "Validity of magnetotransport detection of skyrmions in epitaxial srruo 3 heterostructures," Phys. Rev. Materials 4, 054402 (2020).

[29] K.S. Denisov, I.V. Rozhansky, N.S. Averkiev, and E. Lähderanta, "A nontrivial crossover in topological Hall effect regimes," Scientific Reports 7, 1-11 (2017).

[30] I. Dzyaloshinsky, "A thermodynamic theory of "weak" ferromagnetism of antiferromagnetics," Journal of Physics and Chemistry of Solids 4, 241-255 (1958).

[31] T. Moriya, "Anisotropic superexchange interaction and weak ferromagnetism," Physical review 120, 91 (1960).

[32] J. Bouaziz, M. dos Santos Dias, A. Ziane, M. Benakki, S. Blügel, and S. Lounis, "Chiral magnetism of magnetic adatoms generated by Rashba electrons," New Journal of Physics 19, 023010 (2017).

[33] J. Fransson, "Inelastic-impurity-scattering-induced spin texture and topological transitions in surface electron waves," Phys. Rev. B 92, 125405 (2015).

[34] K. S. Denisov, I. V. Rozhansky, N. S. Averkiev, and E. Lähderanta, "Electron Scattering on a Magnetic Skyrmion in the Nonadiabatic Approximation," Phys. Rev. Lett. 117, 027202 (2016).

[35] F. R. Lux, F. Freimuth, S. Blügel, and Y. Mokrousov, "Chiral Hall Effect in Noncollinear Magnets from a Cyclic Cohomology Approach," Phys. Rev. Lett. 124, 096602 (2020).

[36] G. A. Fiete and E. J. Heller, "Colloquium: Theory of quantum corrals and quantum mirages," Rev. Mod. Phys. 75, 933-948 (2003).

[37] J. Bouaziz, S. Lounis, S. Blügel, and H. Ishida, "Microscopic theory of the residual surface resistivity of Rashba electrons," Phys. Rev. B 94, 045433 (2016).

[38] See Supplemental Material [url] for details on the multiple scattering expansions, forms of the Green function and the micromagnetic limit. The Supplemental Material also includes Refs.[69-76].

[39] Y. A. Bychkov and E. I. Rashba, "Oscillatory effects and the magnetic susceptibility of carriers in inversion layers," Journal of physics C: Solid state physics 17, 6039 (1984).

[40] J. Bouaziz, M. dos Santos Dias, F. S. M. Guimarães, S. Blügel, and S. Lounis, "Impurity-induced orbital magnetization in a Rashba electron gas," Phys. Rev. B 98, 125420 (2018).

[41] J. Sinova, S. O. Valenzuela, J. Wunderlich, C. H. Back, and T. Jungwirth, "Spin Hall effects," Rev. Mod. Phys. 87, 1213-1260 (2015).

[42] M. dos Santos Dias, J. Bouaziz, M. Bouhassoune, S. Blügel, and S. Lounis, "Chirality-driven orbital magnetic moments as a new probe for topological magnetic structures," Nature Communications 7, 1-6 (2016).

[43] F. Reinert, G. Nicolay, S. Schmidt, D. Ehm, and S. Hüfner, "Direct measurements of the L-gap surface states on the (111) face of noble metals by photoelectron spectroscopy," Phys. Rev. B 63, 115415 (2001).

[44] K. Ishizaka, M.S. Bahramy, H. Murakawa, M. Sakano, T. Shimojima, T. Sonobe, K. Koizumi, S. Shin, H. Miyahara, A. Kimura, K. Miyamoto, T. Okuda, H. Namatame, M. Taniguchi, R. Arita, N. Nagaosa, K. Kobayashi, Y. Murakami, R. Kumai, Y. Kaneko, Onose Y., and Tokura Y., "Giant Rashba-type spin splitting in bulk BiTeI," Nature Materials 10, 521-526 (2011).

[45] C. R. Ast, J. Henk, A. Ernst, L. Moreschini, M. C. Falub, D. Pacilé, P. Bruno, K. Kern, and M. Grioni, "Giant Spin Splitting through Surface Alloying," Phys. Rev. Lett. 98, 186807 (2007).

[46] E. Frantzeskakis, A. Crepaldi, S. Pons, K. Kern, and M. Grioni, "Tuning the giant Rashba effect on a BiAg2 surface alloy: Two different approaches," Journal of Electron Spectroscopy and Related Phenomena 181, 88-95 (2010).

[47] R. Winkler, S. Papadakis, E. De Poortere, and M. Shayegan, Spin-Orbit Coupling in Two-Dimensional Electron and Hole Systems, Vol. 41 (Springer, 2003).

[48] X. Wan, A. M. Turner, A. Vishwanath, and S. Y. Savrasov, "Topological semimetal and Fermi-arc surface states in the electronic structure of pyrochlore iridates," 
Phys. Rev. B 83, 205101 (2011).

[49] C. Bradley and A. Cracknell, The mathematical theory of symmetry in solids: representation theory for point groups and space groups (Oxford University Press, 2009).

[50] L. Šmejkal and T. Jungwirth, "Symmetry and topology in antiferromagnetic spintronics," in Topology in Magnetism (Springer, 2018) pp. 267-298.

[51] M. Seemann, D. Ködderitzsch, S. Wimmer, and H. Ebert, "Symmetry-imposed shape of linear response tensors," Phys. Rev. B 92, 155138 (2015).

[52] T. Kikuchi, T. Koretsune, R. Arita, and G. Tatara, "Dzyaloshinskii-Moriya interaction as a consequence of a Doppler shift due to spin-orbit-induced intrinsic spin current," Phys. Rev. Lett 116, 247201 (2016).

[53] K-W. Kim, H-W. Lee, K-J. Lee, and M. D. Stiles, "Chirality from interfacial spin-orbit coupling effects in magnetic bilayers," Phys. Rev. Lett 111, 216601 (2013).

[54] F. Freimuth, S. Blügel, and Y. Mokrousov, "Relation of the Dzyaloshinskii-Moriya interaction to spin currents and to the spin-orbit field," Phys. Rev. B 96, 054403 (2017).

[55] B. Göbel, I. Mertig, and O. A. Tretiakov, "Beyond skyrmions: Review and perspectives of alternative magnetic quasiparticles," arXiv preprint arXiv:2005.01390 (2020).

[56] N. Nagaosa and Y. Tokura, "Topological properties and dynamics of magnetic skyrmions," Nature Nanotechnology $\mathbf{8}, 899$ (2013).

[57] S-Z. Lin, A. Saxena, and C. D. Batista, "Skyrmion fractionalization and merons in chiral magnets with easyplane anisotropy," Phys. Rev. B 91, 224407 (2015).

[58] N. Romming, C. Hanneken, M. Menzel, J. E. Bickel, B. Wolter, K. von Bergmann, A. Kubetzka, and R. Wiesendanger, "Writing and deleting single magnetic skyrmions," Science 341, 636-639 (2013).

[59] D. M. Crum, M. Bouhassoune, J. Bouaziz, B. Schweflinghaus, S. Blügel, and S. Lounis, "Perpendicular reading of single confined magnetic skyrmions," Nature Communications 6, 1-8 (2015).

[60] I. L. Fernandes, J. Bouaziz, S. Blügel, and S. Lounis, "Universality of defect-skyrmion interaction profiles," Nature Communications 9, 1-7 (2018).

[61] T. Schulz, R. Ritz, A. Bauer, M. Halder, M. Wagner, C. Franz, Christian P., Karin Everschor, M. Garst, and A. Rosch, "Emergent electrodynamics of skyrmions in a chiral magnet," Nature Physics 8, 301-304 (2012).

[62] P. K. Muduli, K.-J. Friedland, J. Herfort, H.-P. Schönherr, and K. H. Ploog, "Antisymmetric contribution to the planar Hall effect of $\mathrm{Fe}_{3} \mathrm{Si}$ films grown on GaAs(113)A substrates," Phys. Rev. B 72, 104430 (2005).
[63] R. Streubel, P. Fischer, F. Kronast, V. P. Kravchuk, D. D. Sheka, Y. Gaididei, O. G. Schmidt, and D. Makarov, "Magnetism in curved geometries," Journal of Physics D: Applied Physics 49, 363001 (2016).

[64] F. N. Rybakov, N. S. Kiselev, A. B. Borisov, L. Döring, C. Melcher, and S. Blügel, "Magnetic hopfions in solids," arXiv preprint arXiv:1904.00250 (2019).

[65] F. R. Lux, F. Freimuth, S. Blügel, and Y. Mokrousov, "Engineering chiral and topological orbital magnetism of domain walls and skyrmions," Communications Physics 1, 1-8 (2018).

[66] A. K. Nayak, J. E. Fischer, Y. Sun, B. Yan, J. Karel, A. C. Komarek, C. Shekhar, N. Kumar, W. Schnelle, J. Kübler, C. Felser, and S. S. P. Parkins, "Large anomalous Hall effect driven by a nonvanishing Berry curvature in the noncolinear antiferromagnet Mn3Ge," Science advances 2, e1501870 (2016).

[67] O. Busch, B. Göbel, and I. Mertig, "Microscopic origin of the anomalous Hall effect in noncollinear kagome magnets," Phys. Rev. Research 2, 033112 (2020).

[68] S. Grytsiuk, J-P. Hanke, M. Hoffmann, J. Bouaziz, O. Gomonay, G. Bihlmayer, S. Lounis, Y. Mokrousov, and S. Blügel, "Topological-chiral magnetic interactions driven by emergent orbital magnetism," Nature Communications 11, 1-7 (2020).

[69] H. Friedrich, Scattering theory, Vol. 872 (Springer, 2013).

[70] J. Zabloudil, R. Hammerling, L. Szunyogh, and P. Weinberger, Electron Scattering in Solid Matter: a theoretical and computational treatise, Vol. 147 (Springer Science \& Business Media, 2006).

[71] S. Lounis, A. Bringer, and S. Blügel, "Magnetic Adatom Induced Skyrmion-Like Spin Texture in Surface Electron Waves," Phys. Rev. Lett. 108, 207202 (2012).

[72] J-P. Jan, "Galvamomagnetic and thermomagnetic effects in metals," Solid State Physics, 5, 1-96 (1957).

[73] M. Trushin, K. Výborný, P. Moraczewski, A. A. Kovalev, J. Schliemann, and T. Jungwirth, "Anisotropic magnetoresistance of spin-orbit coupled carriers scattered from polarized magnetic impurities," Phys. Rev. B 80, 134405 (2009).

[74] M. L. Cohen and S. G. Louie, Fundamentals of condensed matter physics (Cambridge University Press, 2016).

[75] Jochen Brüning, Vladimir Geyler, and Konstantin Pankrashkin, "Explicit green functions for spin-orbit hamiltonians," Journal of Physics A: Mathematical and Theoretical 40, F697-F704 (2007).

[76] Hao Yang, Yan Sun, Yang Zhang, Wu-Jun Shi, Stuart S P Parkin, and Binghai Yan, "Topological weyl semimetals in the chiral antiferromagnetic materials mn3ge and mn3sn," New Journal of Physics 19, 015008 (2017). 